\title{
Clinical utility of a type 4 portable device for in-home screening of sleep disordered breathing
}

\author{
Yuriko Yamada ${ }^{1,2}$, Satoshi Kasagi ${ }^{2}$, Haruko Tamura ${ }^{1}$, Fusae Kawana ${ }^{3,4}$, Yasuhiro Tomita ${ }^{2}$, Koji Narui ${ }^{2}$, \\ Takatoshi Kasai ${ }^{2,3,4,5}$
}

${ }^{1}$ Clinical Physiology, Toranomon Hospital, Tokyo, Japan; ${ }^{2}$ Department of Sleep Respiratory Medicine, Toranomon Hospital, Tokyo, Japan; ${ }^{3}$ Cardiovascular Respiratory Sleep Medicine, Juntendo University Graduate School of Medicine, Tokyo, Japan; ${ }^{4}$ Sleep and Sleep Disordered Breathing Center, Juntendo University Hospital, Tokyo, Japan; ${ }^{5}$ Department of Cardiovascular Medicine, Juntendo University Graduate School of Medicine, Tokyo, Japan

Contributions: (I) Conception and design: Y Yamada, S Kasagi, T Kasai; (II) Administrative support: F Kawana, Y Tomita, K Narui; (III) Provision of study materials or patients: S Kasagi, K Narui; (IV) Collection and assembly of data: Y Yamada, S Kasagi; (V) Data analysis and interpretation: S Kasagi, T Kasai; (VI) Manuscript writing: All authors; (VII) Final approval of manuscript: All authors.

Correspondence to: Satoshi Kasagi, MD, PhD. Department of Sleep Respiratory Medicine, Toranomon Hospital, 2-2-2 Toranomon, Minato-ku, Tokyo 105-8470, Japan. Email: s-kasagi@toranomon.gr.jp.

Background: Portable monitoring devices have been developed for in-home screening and to aid in the diagnosis of sleep disordered breathing (SDB) while increasing accessibility and reducing costs. Although there are many different devices available in the market, most have not undergone rigorous validation. Therefore, although such devices are promising, more research on their clinical utility is necessary. The purpose of this study was to assess the clinical utility of a type 4 home sleep apnea test (HSAT) as an in-home screening for SDB.

Methods: We investigated consecutive subjects who underwent in-laboratory overnight polysomnography following in-home screening using HSAT. We evaluated the correlation between apnea-hypopnea index (AHI) by in-laboratory overnight polysomnography and by HSAT and evaluated the sensitivity and specificity for $\mathrm{AHI} \geq 5$ and $\mathrm{AHI} \geq 30$ by the receiver operating characteristic (ROC) analysis.

Results: Finally, data of 387 participants $(86.8 \%$ men, mean age $55.3 \pm 13.3$ years and body mass index $25.1 \pm 4.1 \mathrm{~kg} / \mathrm{m}^{2}$ ) were assessed. In all patients, AHI by HSAT correlated significantly with AHI by polysomnography $(\mathrm{r}=0.670, \mathrm{P}<0.001)$. The area under curves of $\mathrm{ROC}$ for $\mathrm{AHI} \geq 5$ and $\mathrm{AHI} \geq 30$ were $0.854 \pm 0.029$ and $0.841 \pm 0.022$, respectively. The best cut-off of AHI by HSAT for detecting AHI by polysomnography $\geq 5$ was 10.3 events/h (sensitivity, $82.8 \%$; and specificity, $76.0 \%$ ), and AHI by HSAT for detecting AHI by polysomnography $\geq 30$ was 24.5 events/h (sensitivity, $75.8 \%$; and specificity, $80.4 \%$ ).

Conclusions: This type 4 HSAT may have potential as a screening tool for SDB and thus have sufficient clinical utility.

Keywords: Sleep disordered breathing (SDB); apnea-hypopnea index (AHI); home sleep apnea test (HSAT); inlaboratory overnight polysomnography

Submitted Feb 12, 2020. Accepted for publication Jul 31, 2020.

doi: $10.21037 /$ apm-20-384

View this article at: http://dx.doi.org/10.21037/apm-20-384 


\section{Introduction}

Sleep disordered breathing (SDB) has been widely recognized as a prevalent disorder and a recent update of the Wisconsin cohort study reported a disturbing increase in estimated prevalence of obstructive sleep apnea (OSA) over the past two decades, in association with an aging population and increasing obesity. However, there might be many undiagnosed patients (1-5). Although in-laboratory overnight polysomnography is regarded as a gold standard of examination for OSA, it is costly, labor-intensive, and has limited accessibility, and often has long waiting times $(6,7)$. To resolve these issues, many laboratories have prompted a search for alternatives that could facilitate the diagnosis of OSA $(8,9)$. Therefore, many types of portable monitoring devices have been developed for in-home screening and diagnosis of OSA.

In general, devices and techniques for detecting SDB are classified into four types (10). Type 1 indicates attended full polysomnography equipped with a minimum of seven channels, generally including electroencephalogram, electrooculogram, chin electromyogram, pulse rate or electrocardiogram, respiratory effort, airflow, and oxygen saturation in the laboratory. Type 2 indicates a minimum of seven channels in an unattended setting. Type 3 indicates test using a minimum of four channels, including respiratory movement, airflow, heart rate or electrocardiogram, and oxygen saturation. Type 4 indicates a test with a single or two parameters, usually using oxygen saturation plus airflow or pulse rate. Currently, Type 3 and 4 devices are called portable cardio-respiratory monitors or home sleep apnea test (HSAT) and can be used as a screening tool for OSA or as an aid for diagnosis of OSA for patients with a high pretest probability of moderate to severe OSA (11-13). Thus, many HSAT devices have been developed for clinical use. Among them, Apnomonitor ${ }^{\circledR}$ (Chest M.I.Inc., Tokyo, Japan), a Type 4 HSAT device, developed in Japan, has been used widely, and is one of the most popular HSAT devices. However, although some data have shown the utility of Apnomonitor ${ }^{\circledR}$ for screening of OSA, clinical utility of the current Apnomonitor $^{\circledR}$ series device, Apnomonitor Mini ${ }^{\circledR}$, is not verified $(14,15)$. Thus, the purpose of this study was to assess the clinical utility of the Apnomonitor Mini ${ }^{\circledR}$.

We present the following article in accordance with the
STROBE reporting checklist (available at http://dx.doi. org/10.21037/apm-20-384).

\section{Methods}

\section{Subjects}

Three hundred and eighty-seven consecutive patients who underwent overnight polysomnography (Toranomon Hospital, Tokyo, Japan) following overnight HSAT from January 2009 to June 2010 and finally diagnosed with OSA by polysomnography were enrolled. HSAT had been done within 1 month prior to the in-laboratory polysomnography. Exclusion criteria were: (I) patients who underwent split night study for the diagnostic polysomnography; (II) patients with incomplete HSAT (i.e., loss of pressure sensor signal or oximetry signal $\geq 50 \%$ of total recording); (III) patients with predominant central sleep apnea by polysomnography; and (IV) patients receiving oxygen therapy for the respiratory failure or mechanical ventilation. The trial was conducted in accordance with the Declaration of Helsinki (as revised in 2013). The Toranomon Hospital Ethics Committee approved this study (No. 1877), and all participants provided written informed consent.

\section{HSAT device}

We performed the sleep test using a type 4 HSAT device, Apnomonitor Mini ${ }^{\circledR}$ (CHEST M.I. Inc., Tokyo, Japan), which is easy to use and small, and comprises a combined thermistor and nasal pressure transducer signals, a finger pulse oximeter, a microphone to monitor tracheal sounds, a position detector and the integrative unit (Figure 1). Each sensor was connected to the integrative unit to record the following parameters: airflow signal, snoring, percutaneous saturation $\left(\mathrm{SpO}_{2}\right)$, pulse rate, and body position.

The Apnomonitor Mini ${ }^{\circledR}$ detected apneas or hypopneas by the waveform peak of respiration when the reference waveform was recognized as the averaged amplitude during the 2-min interval just prior to the monitoring. Apnea was defined as a reduction in airflow through the nose greater than $75 \%$ of baseline, lasting at least 10 seconds. Hypopnea was defined as a reduction of airflow or in thoracoabdominal movement greater than $50 \%$ of baseline, lasting at least 


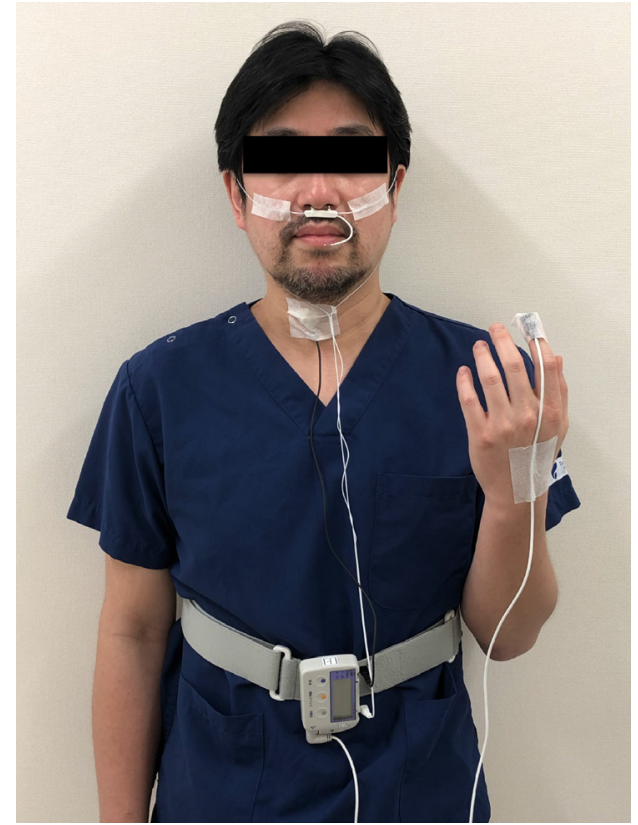

Figure 1 Picture of home sleep apnea test.

10 seconds. These respiratory events were automatically scored, and the apnea-hypopnea index (AHI) was calculated as the frequency of apneas and hypopneas per hour of recording (AHI-HSAT). Minimum levels of oxyhemoglobin saturation $\left(\mathrm{SpO}_{2}\right)$ and the number of desaturations per hour of recording, expressed as the oxygen desaturation index (ODI), were also computed. In the present study, desaturations were defined as $\geq 4 \%$ and $\geq 3 \%$ decrease in the saturation level (4\% ODI and 3\% ODI, respectively). The percentage of time that $\mathrm{SpO}_{2}$ was less than $90 \%$ was also expressed as a percentage of total recording time. After acquisition, data were processed using a personal computer. An experienced technician, blinded to the information regarding the subjects, performed the scoring manually. Subsequently, a sleep specialist, also blinded to the subject information, double-checked scored data.

\section{Polysomnography}

Polysomnography was performed using a digital polygraph (SomnoStar a Sleep System; Sensor Medics Corp., Yorba Linda, CA) monitored by the sleep technicians in our sleep center (Toranomon Hospital, Tokyo, Japan). The accepted definitions and scoring methods for the diagnostic polysomnography i.e., apnea was defined as a cessation of airflow for at least 10 seconds; hypopnea was defined as a reduction of airflow by more than $50 \%$ for at least 10 seconds or discernible reduction of airflow with desaturation or an arousal (16-18). In each case, the respiratory events were manually scored; the following parameters were obtained from the polysomnography and the AHI was calculated per hour of sleep (AHI-PSG). All HSAT using Apnomonitor Mini ${ }^{\circledR}$ have been performed on a different day, before polysomnography, within 1 month. An experienced technician who was blinded to the information regarding HSAT manually scored all polysomnography recordings. Subsequently, a sleep specialist who was blinded to the information regarding HSAT double-checked scored data.

\section{Statistical analysis}

Data are expressed as the mean \pm standard deviation (SD) or median [interquartile range (IQR)] for continuous variables. Data derived from HSAT devices were compared with those derived from polysomnography using the $t$-test, Wilcoxon rank-sum test, correlation analysis, and Bland and Altman plots (19). The Bland and Altman plot is a graphical representation of the observed differences between AHIPSG and AHI-HSAT. Sensitivity, specificity and accuracy were calculated according to the value of AHI-PSG $\geq 5$ and $\geq 30$. Accuracy was calculated as total number of subjects in true positives plus total number of subjects in true negatives divided by the total number of all subjects. The best cut-off values of AHI-HSAT for detecting AHI-PSG $\geq 5$ and $\geq 30$ was identified by a receiver operating characteristic (ROC) curve analysis. In the ROC curve, the higher the resulting area under the curve (AUC), the higher the discriminatory power of the cut-off values. In addition, we performed subgroup analyses including severity of OSA category (severe OSA or not, cutoff, $\mathrm{AHI}<\mathrm{or} \geq 30$ ) and presence or absence of obesity [body mass index (BMI) $<$ or $\geq 25 \mathrm{~kg} / \mathrm{m}^{2}$ ]. The first-order interactions in regression models were examined by entering interaction terms between the AHIHSAT and the abovementioned subgroup variables. The $P$ value of less than 0.05 was taken as the level of statistical significance. Statistical analysis was performed using SPSS statistical software (SPSS 25.0; SPSS Inc., Chicago, IL, USA).

\section{Results}

Overall, we enrolled 387 patients from January 2009 to June 2010. The average BMI was $25.1 \pm 4.1 \mathrm{~kg} / \mathrm{m}^{2}$. The characteristics 

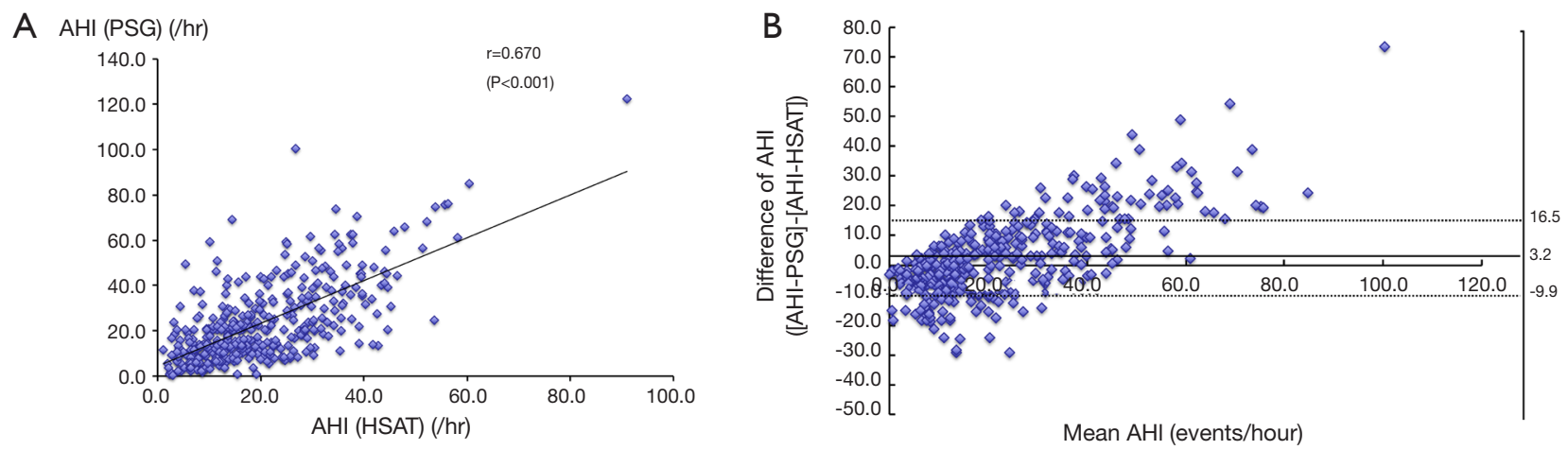

Figure 2 A comparisons of AHI derived from the polysomnography (AHI-PSG) and AHI from home sleep apnea test (AHI-HSAT) are shown. (A) Scatter plots of the AHI derived from the polysomnography (AHI-PSG) and the AHI from home sleep apnea test (AHI-HSAT) in all patients; (B) Bland-Altman plots according to the AHI. The Y axis indicates the difference between the AHI-PSG and AHI-HSAT [(AHI-PSG)-(AHI-HSAT)]. The $\mathrm{X}$ axis indicates the mean values. The solid line represents the mean difference between AHI-PSG and AHI-HSAT; the dashed lines represent the limit of agreement ( $\pm 2 \mathrm{SD})$. AHI, apnea-hypopnea index.

and findings of diagnostic polysomnography are shown in Tables 1 and 2. The mean Epworth Sleepiness Scale score was 9.8 \pm 5.3. The mean AHI-PSG was $23.5 \pm 17.7$ events/h, whereas the mean AHI-HSAT was $20.2 \pm 12.6$ events/h. There was moderate and significant correlation between AHI-HSAT and AHI-PSG (Figure 2A). Bland and Altman plot for AHI-HSAT and AHI-PSG indicated acceptable agreement between them (i.e., mean difference, +3.2 events/h, and the limits of agreement, -9.9 to +16.5 ; Figure $2 B)$. Area under ROC curves were $0.842(\mathrm{P}<0.001)$ for AHIPSG $\geq 5$ and $0.835(\mathrm{P}<0.001)$ for AHI-PSG $\geq 30$. The best cut-off of AHI-HSAT for detecting AHI-PSG $\geq 5$ was 10.3 events/h (a sensitivity of $82.8 \%$ and a specificity of $76.0 \%$ ), and for detecting AHI-PSG $\geq 30$ was 24.5 events/h (a sensitivity of $75.8 \%$ and a specificity of $80.4 \%$ ). When using an $\mathrm{AHI} \geq 5$ cutoff, accuracy in all patients was $89.7 \%$, whereas when using an $\mathrm{AHI} \geq 30$ cutoff, accuracy in all patients was $80.6 \%$.

Correlation between AHI-HSAT and AHI-PSG was more prominent in patients with severe OSA compared with those without severe OSA $(\mathrm{P}=0.003$ for interaction, Figure 3). Correlations between AHI-HSAT and AHI-PSG were similar in patients with and without obesity $(\mathrm{P}=0.063$ for interaction, Figure 4).

\section{Discussion}

These findings provide several novel insights into the clinical utility of the current Type-4 HSAT device,
Apnomonitor $\mathrm{Mini}^{\circledR}$, which is easy to use, small and widely used in Japan. First, AHI-HSAT by Apnomonitor Mini ${ }^{\circledR}$ correlated significantly with AHI-PSG even though they were performed separately (within a gap of 1-month). Second, the best cut off AHI-HSAT value for detecting AHI-PSG $\geq 5$ was 10.3 events/h, and for detecting AHIPSG $\geq 30$ was 24.5 events/h, determined with acceptable sensitivity and specificity. Finally, correlations between AHI-HSAT and AHI-PSG remain significant in severe/ non-severe OSA or obese/non-obese subgroups although slightly better correlations were observed in patients with severe OSA compared with their counterparts. Taken together, screening using Apnomonitor Mini ${ }^{\circledR}$ provides useful information as HSAT, and AHI-HSAT $\geq 10$ events/h and AHI-HSAT $\geq 24$ events/h should be applied to screen even mild OSA and severe OSA, respectively. In addition, Apnomonitor Mini ${ }^{\circledR}$ can be used particularly in patients with high pretest probability for severe OSA.

Although in-laboratory overnight polysomnography is the standard diagnostic test for OSA, many patients suspected of having sleep apnea may not be able to undergo this test because of its high cost, limited access, and long waiting time $(6,7)$. In addition, patients may be unwilling to undergo an in-laboratory overnight polysomnography without objective data suspecting the presence of OSA. Currently, to obtain such objective data, Type 3 and 4 HSAT devices are used in Japan, which may be helpful as a screening tool for symptomatic populations, for either OSA or severe OSA. Usually, measurement error is inevitable in 

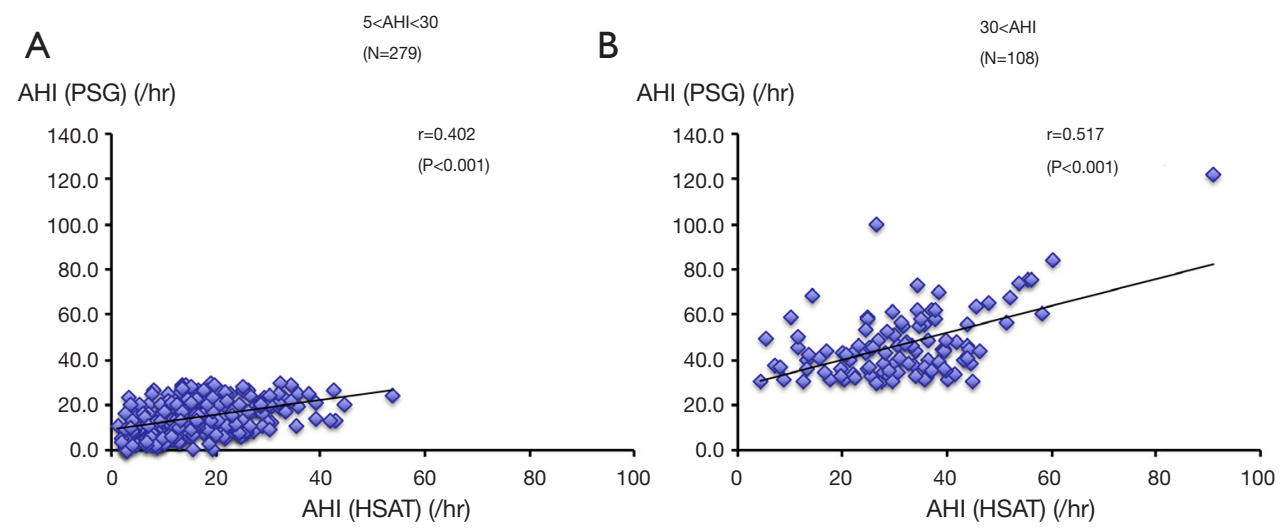

Figure 3 Scatter plots of the AHI derived from the polysomnography (AHI-PSG) and the AHI from home sleep apnea test (AHI-HSAT) in mild to moderate OSA and severe OSA. AHI, apnea-hypopnea index; OSA, obstructive sleep apnea.
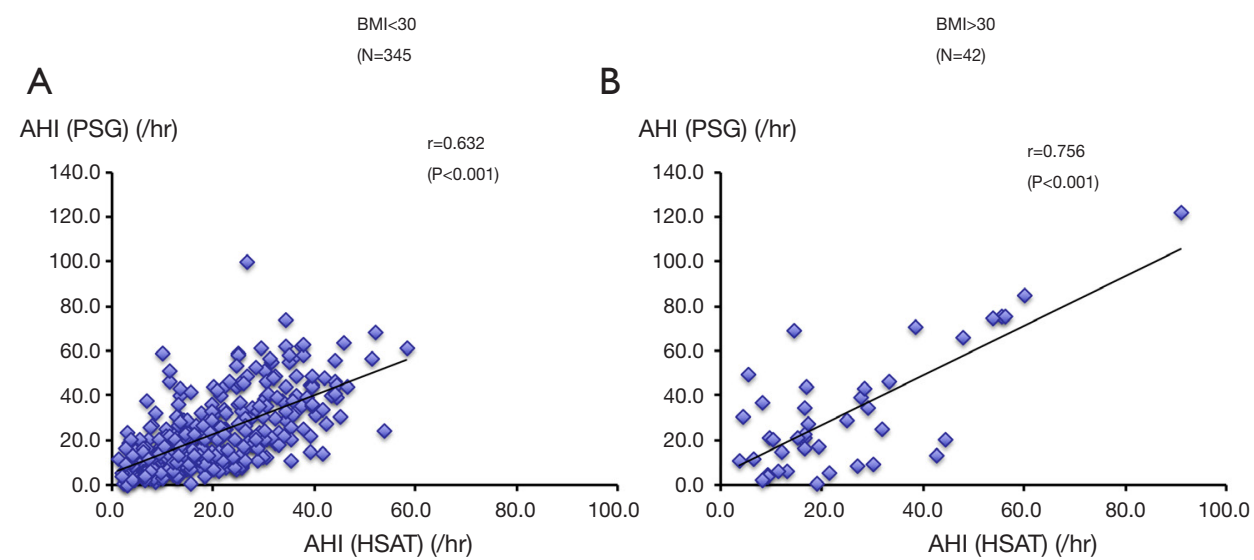

Figure 4 Scatter plots of the AHI derived from the polysomnography (AHI-PSG) and the AHI from home sleep apnea test (AHI-HSAT) in non-obese and obese OSA. AHI, apnea-hypopnea index; OSA, obstructive sleep apnea.

HSAT, compared to polysomnography, as standard sleep staging channels are not typically monitored in HSAT, which results in use of recording time rather than sleep time to define the denominator of the respiratory event index. HSAT devices are unable to detect hypopneas only associated with cortical arousals, which are included in the recommended AHI scoring rule in the American Academy of Sleep Medicine (AASM) Scoring Manual (18,20,21). To verify the accuracy of HSAT, diagnostic guideline has been revised as incorporating evidence regarding the accuracy of HSAT for diagnosing OSA in 2017 AASM clinical practice guideline (16). In the AASM clinical practice guideline, it is recommended that HSAT with a technically adequate device can be used for the diagnosis of OSA in uncomplicated adult patients presenting with signs and symptoms that indicate an increased risk of moderate to severe OSA. In studies evaluating the performance of Type 3 HSAT devices against polysomnography, when using an $\mathrm{AHI} \geq 5$ cutoff, accuracy in a high-risk population ranged from $84 \%$ to $91 \%$, whereas in a low-risk population accuracy ranged from $70 \%$ to $78 \%$. When using a cutoff of AHI $\geq 30$, the accuracy of the devices in the high-risk population was $88 \%$ (95\% CI: $81 \%$ to $94 \%$ ). Other studies evaluating the performance of Type 4 HSAT devices against polysomnography, when using an AHI $\geq 5$ cutoff, accuracy in a high-risk population ranged from $81 \%$ to $93 \%$, whereas in a low-risk population accuracy ranged from $77 \%$ to $88 \%$. When using a cutoff of $\mathrm{AHI} \geq 30$, the accuracy of the devices in the high-risk population ranged from $71 \%$ to $90 \%$, whereas in a low-risk population accuracy ranged from $88 \%$ to $91 \%$. Our results 
Table 1 Demographic characteristics and comorbidities

\begin{tabular}{ll}
\hline Demographic characteristics & Results \\
\hline Age $(\mathrm{y})$ & $55.3 \pm 13.3$ \\
$\mathrm{BMI}, \mathrm{kg} / \mathrm{m}^{2}$ & $25.1 \pm 4.1$ \\
$\mathrm{ESS}$ & $9.8 \pm 5.3$ \\
$\mathrm{AHI}, \mathrm{h}$ (home sleep apnea test) & $20.2 \pm 12.5$ \\
Hypertension, $\mathrm{n}(\%)$ & $186(48.1)$ \\
Hyperlipidemia, $\mathrm{n}(\%)$ & $130(33.6)$ \\
Diabetes, $\mathrm{n}(\%)$ & $81(20.9)$ \\
Arrhythmia, $\mathrm{n}(\%)$ & $15(3.9)$ \\
\hline
\end{tabular}

ESS, Epworth Sleepiness Scale; AHI, apnea-hypopnea index.

Table 2 Findings of diagnostic polysomnography

\begin{tabular}{ll}
\hline Polysomnography & Results \\
\hline TST, min & $347.2 \pm 63.0$ \\
AHI, /h & $23.5 \pm 17.7$ \\
\%Obstructive, \% & $92.1 \pm 29.0$ \\
\%Central, \% & $5.8 \pm 11.2$ \\
\%Mixed, \% & $2.5 \pm 8.7$ \\
Min SpO,$\%$ & $81.5 \pm 7.6$ \\
Arousal index, /h & $34.7 \pm 15.6$ \\
Sleep stage, \%TST & \\
Stage N1, \%TST & $31.0 \pm 14.4$ \\
Stage N2, \%TST & $46.8 \pm 11.6$ \\
Stage N3, \%TST & $11.4 \pm 7.9$ \\
Stage R, \%TST & $10.8 \pm 5.3$ \\
PLM index, /h & $10.6 \pm 21.5$ \\
\hline
\end{tabular}

Data are expressed as the mean \pm standard deviation (SD) or median [interquartile range (IQR)]. BMI, body mass index; ESS, Epworth Sleepiness Scale; AHI, apnea-hypopnea index; TST, total sleep time; SpO2, arterial oxygen saturation; PLM, periodic limb movement.

were comparable to these reports (20,22-25).

The lack of sleep variables in type 4 device studies in patients with suspected OSA has several potentially relevant limitations. Thus, there is a possibility that AHIHSAT might be underestimated AHI-PSG as mentioned earlier. Indeed, in the present study, mean AHI-HSAT was smaller than mean AHI-PSG. However, mean difference in
AHI was only 3.2 with acceptable limit of agreement, and acceptable specificity and sensitivity to detect AHI-PSG $\geq 30$ events/h. Considering that correlation between AHIHSAT and AHI-PSG was more prominent in patients with severe OSA in the present study, patients with severe OSA based on AHI-PSG can be sufficiently detected by HSAT using Apnomonitor Mini ${ }^{\circledR}$. In 2017 AASM clinical practice guideline for HSAT recommended that HSAT might be uses as an alternative to polysomnography for the diagnosis of OSA only in patients with a high pretest probability of moderate to severe OSA (16). Our data were in line with this.

In contrast, correlation coefficient between AHIHSAT and AHI-PSG was lower in patients without severe OSA, and the best cutoff AHI-HSAT value to detect AHIPSG $\geq 5$ events/h was approximately 10 events/h. Thus, there is a possibility that HSAT using Apnomonitor Mini ${ }^{\circledR}$ may overestimate AHI-PSG in patients with an AHIPSG $<30$ events/h, and this should be taken into account. Nevertheless, AHI-HSAT by Apnomonitor Mini ${ }^{\circledR}$ had acceptable specificity and sensitivity for detecting even AHI-PSG $\geq 5$ events/h.

More work is needed to determine the optimal duration for diagnostic testing of polysomnography following inhome screening using HSAT. In several studies evaluating the performance of Type 4 HSAT devices against polysomnography, the duration of diagnostic testing of polysomnography following in-home screening using HSAT was within 2 weeks. It is necessary to examine whether the period within 1-month prior affects clinically meaningful outcomes. However, it was considered that the period within 1-month prior did not affect clinically meaningful outcomes as our results show that Apnomonitor $\mathrm{Mini}^{\circledR}$ had acceptable specificity and sensitivity for detecting OSA.

Despite no significant interaction regarding obese/nonobese subgroups and AHI correlation, positive correlation was more prominent in obese patients (i.e., BMI $\geq 30$ ) compared to non-obese patients (i.e., BMI <30). Obesity is one of the important co-existing condition of OSA, and in general, is regarded as a first step for OSA screening (3). Thus, it is important for obese subjects to determine whether they really have severe OSA requiring treatment. To this end, despite no significant interaction between obese/no-obese patients, a more prominent correlation between AHI-HSAT and AHI-PSG was observed in this study. Because obese subjects are more likely to have comorbidities such as hypertension and cardiovascular 
disease, and because reduction of blood pressure, and improvement of some cardiovascular parameters can be observed by the treatment of OSA, their OSA should be identified and be referred for immediate treatment. Physicians should recognize the clinical utility of Apnomonitor Mini ${ }^{\circledR}$ for screening of OSA as shown in the present study.

\section{Conclusions}

In conclusion, the clinical utility of Type 4 HSAT device Apnomonitor $\mathrm{Mini}^{\circledR}$ as a screening tool and aid for diagnosis of OSA has been assessed. Apnomonitor Mini ${ }^{\circledR}$ can be a reliable screening and diagnostic tool for OSA.

\section{Acknowledgments}

Funding: This study was partially supported by the Okinaka Memorial Foundation.

\section{Footnote}

Reporting Checklist: The authors have completed the STROBE reporting checklist. Available at http://dx.doi. org/10.21037/apm-20-384

Data Sharing Statement: Available at http://dx.doi. org/10.21037/apm-20-384

Conflicts of Interest: All authors have completed the ICMJE uniform disclosure form (available at http://dx.doi. org/10.21037/apm-20-384). The authors have no conflicts of interest to declare.

Ethical Statement: The authors are accountable for all aspects of the work in ensuring that questions related to the accuracy or integrity of any part of the work are appropriately investigated and resolved. The trial was conducted in accordance with the Declaration of Helsinki (as revised in 2013). This study was approved by ethics board of the Toranomon Hospital (No. 1877) and informed consent was taken from all the patients.

Open Access Statement: This is an Open Access article distributed in accordance with the Creative Commons Attribution-NonCommercial-NoDerivs 4.0 International License (CC BY-NC-ND 4.0), which permits the noncommercial replication and distribution of the article with the strict proviso that no changes or edits are made and the original work is properly cited (including links to both the formal publication through the relevant DOI and the license). See: https://creativecommons.org/licenses/by-nc-nd/4.0/.

\section{References}

1. Durán J, Esnaola S, Rubio R, et al. Obstructive sleep apnea-hypopnea and related clinical features in a population-based sample of subjects aged 30 to $70 \mathrm{yr}$. Am J Respir Crit Care Med 2001;163:685-9.

2. Somers VK, White DP, Amin R, et al. Sleep apnea and cardiovascular disease: an American Heart Association/ American College Of Cardiology Foundation Scientific Statement from the American Heart Association Council for High Blood Pressure Research Professional Education Committee, Council on Clinical Cardiology, Stroke Council, and Council On Cardiovascular Nursing. In collaboration with the National Heart, Lung, and Blood Institute National Center on Sleep Disorders Research (National Institutes of Health). Circulation 2008;118:1080-111.

3. Peppard PE, Young T, Barnet JH, et al. Increased prevalence of sleep-disordered breathing in adult. Am J Epidemiol 2013;177:1006-14.

4. Young T, Blustein J, Finn L, et al. Sleep-disordered breathing and motor vehicle accidents in a populationbased sample of employed adults. Sleep 1997;20:608-13.

5. Flemons WW, Douglas NJ, Kuna ST, et al. Access to diagnosis and treatment of patients with suspected sleep apnea. Am J Respir Crit Care Med 2004;169:668-72.

6. Sleep-related breathing disorders in adults: recommendations for syndrome definition and measurement techniques in clinical research. The Report of an American Academy of Sleep Medicine Task Force. Sleep 1999;22:667-89.

7. Masa JF, Montserrat JM, Duran J. Spanish Group of Breathing Sleep Disorders: Diagnostic access for sleep apnea in Spain. Am J Respir Crit Care Med 2004;170:195-6.

8. Ballester E, Solans M, Vila X, et al. Evaluation of a portable respiratory recording device for detecting apnoeas and hypopnoeas in subjects from a general population. Eur Respir J 2000;16:123-7.

9. Dingli K, Coleman EL, Vennelle M, et al. Evaluation of a portable device for diagnosting the sleep apnoea/ hypopnoea syndrome. Eur Respir J 2003;21:253-9.

10. Chesson AL Jr, Berry RB, Pack A. Practice parameters 
for the use of portable monitoring devices in the investigation of suspected obstructive sleep apnea in adults A joint project sponsored by the American Academy of Sleep Medicine, the American Thoracic Society and the American Collage of Chest Physicians. Sleep 2003;26:907-13.

11. Collop NA, Anderson WM, Boehlecke B, et al. Clinical guidelines for the use of unattended portable monitors in the diagnosis of obstructive sleep apnea in adult patients Portable monitoring task force of the American Academy of Sleep Medicine. J Clin Sleep Med 2007;3:737-47.

12. Collop NA, Tracy SL, Kapur V, et al. Obstructive sleep apnea devices for out-of center (OOC) testing: technology evaluation. J Clin Sleep Med 2011;7:531-48.

13. Rosen IM, Kirsch DB, Chevin RD, et al. Clinical Use of Home Sleep Apnea Test: An American Academy of Sleep Medicine Position Statement. J Clin Sleep Med 2017;13:1205-7.

14. Hida W, Shidoh C, Miki H, et al. Prevalence of sleep apnea among Japanese industrial workers determined by a portable sleep monitoring system. Respiration 1993;60:332-7.

15. Yagi H, Nakata $S$, Tsuge $H$, et al. Significance of a screening device (Apnomonitor 5) for sleep apnea syndrome. Auris Nasus Larynx 2009;36:176-80.

16. Kapur VK, Auckley DH, Chowdhuri S, et al. Clinical Practice Guideline for Diagnostic Testing for Adult Obstructive Sleep Apnea: An American Academy of Sleep Medicine Clinical Practice Guideline. J Clin Sleep Med 2017;13:479-504.

17. Berry RB, Brooks R, Gamaldo C, et al. AASM Scoring

Cite this article as: Yamada Y, Kasagi S, Tamura H, Kawana F, Tomita Y, Narui K, Kasai T. Clinical utility of a type 4 portable device for in-home screening of sleep disordered breathing. Ann Palliat Med 2020;9(5):2895-2902. doi: 10.21037/apm-20-384
Manual Updates for 2017 (Version 2.4). J Clin Sleep Med 2017;13:665-6.

18. Berry RB, Brooks R, Gamaldo CE, et al. The AASM Manual for the Scoring of Sleep and Associated Events: Rules, Terminology and Technical Specifications. Version 2.3. Darien, IL: American Academy of Sleep Medicine; 2016.

19. Bland JM, Altman DG. Statistical methods for assessing agreement between two methods of clinical measurement. Lancet 1986;1:307-10.

20. Ward KL, McArdle N, Bremner AP, et al. A comprehensive evaluation of a two-channel portable monitor to "rule in" obstructive sleep apnea. J Clin Sleep Med 2015;11:433-44.

21. Wang Y, Teschler T, Weinreich G, et al. [Validation of microMESAM as screening device for sleep disordered breathing]. Pneumologie 2003;57:734-40.

22. Ayappa I, Norman RG, Seelall V, et al. Validation of a selfapplied unattended monitor for sleep disordered breathing. J Clin Sleep Med 2008;4:26-37.

23. de Oliveira ACT, Martinez D, Vasconcelos LFT, et al. Diagnosis of obstructive sleep apnea syndrome and its outcomes with home portable monitoring. Chest 2009;135:330-6.

24. Baltzan MA, Verschelden P, Al-Jahdali H, et al. Accuracy of oximetry with thermistor (OxiFlow) for diagnosis of obstructive sleep apnea and hypopnea. Sleep 2000;23:61-9.

25. Masdeu MJ, Ayappa I, Hwang D, et al. Impact of clinical assessment on use of data from unattended limited monitoring as opposed to full-in lab PSG in sleep disordered breathing. J Clin Sleep Med 2010;6:51-8. 